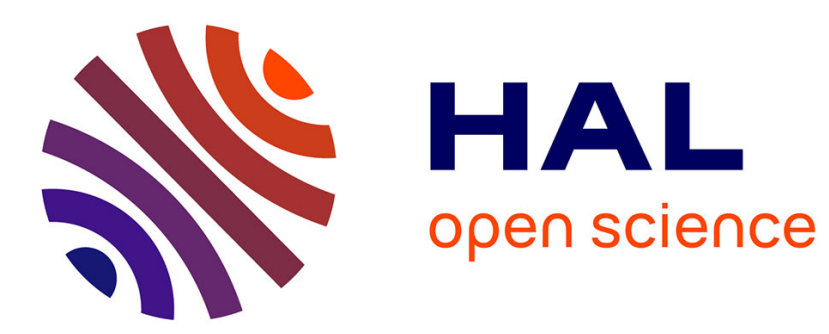

\title{
Flow impact on granular force chains and induced instability
}

\author{
A. Wautier, Stéphane Bonelli, François Nicot
}

\section{To cite this version:}

A. Wautier, Stéphane Bonelli, François Nicot. Flow impact on granular force chains and induced instability. Physical Review E , 2018, 98 (4), pp.042909. 10.1103/PhysRevE.98.042909 . hal-02607878

\section{HAL Id: hal-02607878 \\ https://hal.inrae.fr/hal-02607878}

Submitted on 16 May 2020

HAL is a multi-disciplinary open access archive for the deposit and dissemination of scientific research documents, whether they are published or not. The documents may come from teaching and research institutions in France or abroad, or from public or private research centers.
L'archive ouverte pluridisciplinaire HAL, est destinée au dépôt et à la diffusion de documents scientifiques de niveau recherche, publiés ou non, émanant des établissements d'enseignement et de recherche français ou étrangers, des laboratoires publics ou privés. 


\title{
Flow impact on granular force chains and induced instability
}

\author{
Antoine WAUTIER* \\ AgroParisTech-ENGREF, 19 avenue du Maine, 75732 Paris, France. \\ Irstea UR RECOVER, 3275 Rte Cézanne, CS 40061, 13182 Aix-en-Provence Cedex 5, France. and \\ Université Grenoble Alpes, Irstea, UR ETGR, 2 rue de la Papeterie-BP 76, F-38402 St-Martin-d'Hères, France. \\ Stéphane Bonelli \\ Irstea UR RECOVER, 3275 Rte Cézanne, CS 40061, 13182 Aix-en-Provence Cedex 5, France. \\ François NiCOT \\ Université Grenoble Alpes, Irstea, UR ETGR, 2 rue de la Papeterie-BP 76, F-38402 St-Martin-d'Hères, France.
}

(Dated: September 20, 2018)

\begin{abstract}
With today's fully coupled DEM/PFV simulations, the impact of an internal fluid flow on the mechanical stability of a granular material is assessed at the scale of a three-dimensional representative elementary volume. To isolate the specific impact of the fluid on the contact network, a granular sample in which rattlers have been initially removed is considered while a fluid pressure drop is applied under constant mechanical loading. Although the initial state is reputed to be unstable according to the second order work criterion, it is shown that i) an internal fluid flow is able to trigger the underlying instability on its own and ii) the flow direction has no influence because of local fluid force fluctuations. In addition, the microscale mechanisms occurring during the sample collapse are investigated and it is shown that the fluid perturbation induces a decrease in kinematic constraints around force chains, which is characteristic of a local loss of stability.
\end{abstract}

Keywords: granular material, internal flow, fluid force fluctuations, instabilities, force chains

\section{INTRODUCTION}

Under various circumstances, granular materials subjected to an internal flow can undergo selective erosion of their finest particles. Through fluid/grain interactions, the material's microstructure is then altered, which may result in a change in macroscopic hydraulic and mechanical properties. When this phenomenon occurs for instance in earth dikes and dams, or in water-saturated natural deposits of granular material, a loss of mechanical stability may have dramatic consequences resulting from dike failure or debris flows $[1,2]$.

So far the occurrence of this selective erosion phenomenon (also referred to as suffusion or sand production) has mostly been considered through geometric considerations. Indeed, the notion of internal stability has been introduced in the geomechanics community as the ability of a granular material to self-prevent the loss of the smallest particles under the action of disturbing forces such as seepage or vibration [3]. The notion of self-filtration follows, and most of the criteria used for practical applications are constructed on particle size distributions (PSD) [3-5] or more recently on constriction size distributions (CSD) [6-8]. Only few of them take into account mechanical or hydraulic loading to assess the occurrence of the phenomenon [9-11] and none account for the resulting changes in terms of mechanical stability.

As introduced by Hill [12] within the framework of con-

\footnotetext{
* Corresponding author: antoine.wautier@irstea.fr
}

tinuum mechanics, the second order work criterion has proved to be a very robust criterion to assess the occurrence of divergence instabilities. In particular, this criterion applied to granular materials defines a bifurcation domain formed by the set of stress states in which a suitable incremental loading would result in an inertial transition [13-15]. Using this criterion, the systematic impact of a fluid flow can be investigated in terms of mechanical stability.

For dry cohesionless granular materials, an internal flow does not directly modify the mechanical properties at the contact scale but induces geometrical changes in the microstructure following two kinds of mechanisms. A first change lies in the transport of rattlers, i.e., particles with no contacts with their neighbors in the absence of gravity. Under the action of the fluid, these particles move through the pore space and are either eroded or clogged depending on their size with respect to the size of the pores and constrictions [16-18]. The second flow impact consists in introducing additional forces on the primary skeleton of the granular material [19, 20]. This results in stress redistribution and possibly grain detachment if the existing contact network is forced to rearrange. The first aspect has been widely accounted for through filtration tests $[7,17]$ and the relative impact of clogging and erosion can artificially be obtained by modifying the population of rattlers [21]. The second aspect has been much less investigated, and grain detachment is usually assessed using a Shield criterion at the scale of a single grain and not at the scale of the whole contact network. To isolate the direct impact of a flow on stress transmission, granular samples in which rattlers have been initially removed are considered in this study. From a 


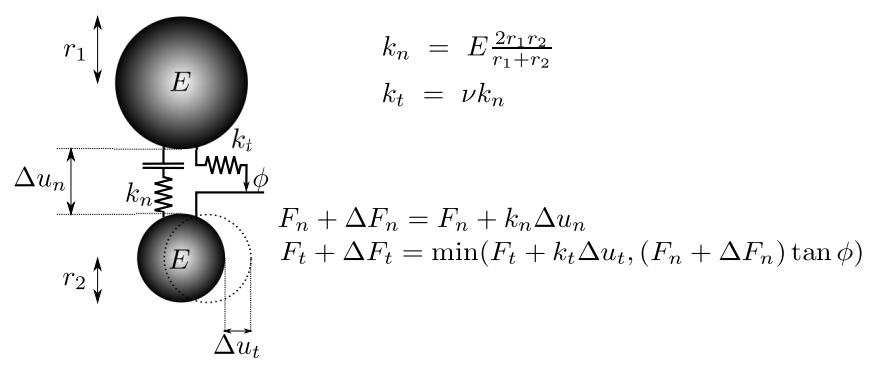

FIG. 1. Elasto-frictional contact law used in DEM simulations.

particular mechanical state identified as unstable with respect to the second order work criterion, the ability of a fluid to trigger underlying instabilities is then assessed using the DEM-PFV fluid grain coupling scheme [22]. Compared to most fluid-grain problems discussed in the literature, this study dares to account for the fluid phase connectivity in $3 \mathrm{D}[10,11,23]$.

From a micromechanical point of view, particular attention is paid to the flow impact on granular force chains constituting the main loadbearing mesostructures in granular materials [24-30].

This paper is organized as follows. In section II, the numerical scheme and the sample preparation procedure are presented. In section III, the ability of a fluid to trigger underlying instabilities is shown and microscale mechanisms are investigated.

\section{NUMERICAL MODELING}

In this section, the discrete element modeling (DEM) adopted in this study is detailed in terms of the used contact law and the fluid/grain coupling scheme. Then the sample generation is briefly explained before introducing the three-dimensional (3D) fluid/grain coupling problem of interest.

\section{A. Contact law}

The micromechanical analysis performed in this paper considers noncohesive granular materials modeled as poly-disperse assemblies of spheres. The interaction between two particles is modeled by the classical elastofrictional contact law proposed by Cundall and Strack [31] and is illustrated in Figure 1. At each contact, a normal force $\boldsymbol{F}_{n}$ and a tangential force $\boldsymbol{F}_{t}$ are defined according to the incremental evolution of the relative displacements $\boldsymbol{\Delta} \boldsymbol{u}_{n}$ and $\boldsymbol{\Delta} \boldsymbol{u}_{t}$ between two contacting particles. The input parameters used in this elasto-frictional contact law are reported in Table I.
TABLE I. Mechanical parameters used in the elasto-frictional contact law implemented in YADE.

\begin{tabular}{ll}
\hline Parameters & Value \\
\hline Density & $3,000 \mathrm{~kg} \cdot \mathrm{m}^{-3}$ \\
Young modulus $(E)$ & $356 \mathrm{MPa}$ \\
Stiffness ratio $(\nu)$ & 0.42 \\
Inter-particle friction angle $(\phi)$ & $35^{\circ}$ \\
Particle-wall friction angle & $0^{\circ}$ \\
Number of particles & 10,000 \\
\hline
\end{tabular}

\section{B. DEM/PFV scheme}

After computing all inter-particle contact forces, the particle displacements induced are integrated based on Newton's second law of motion using the open source code YADE [32] based on a discrete element method (DEM). The fluid grain coupling is accounted for using the pore scale finite volume (PFV) scheme developed by Chareyre et al. [22]. This scheme is based on a discretization of the pore space into a regular Delaunay triangulation. Each pore is thus defined as a tetrahedron with summits corresponding to the center of four grains and is connected to four adjacent pores. The fluid flow problem is then solved directly on this pore network by i) satisfying the fluid flux balance in each pore and ii) assuming Hagen-Poiseuille flows between two adjacent pores. If $\dot{V}_{i}^{f}$ denotes the fluid volume variation of pore $i$ with respect to time, and $q_{i j}$ is the fluid flux between pores $i$ and $j$, the first condition reads $\dot{V}_{i}^{f}=\sum_{j=j_{1}}^{j_{4}} q i j$. By introducing a local conductance $g_{i j}$ between pores $i$ and $j$ the second condition relates the fluid flux $q_{i j}$ to the local pressure gradient as $q_{i j}=g_{i j} \frac{p_{i}-p_{j}}{L_{i j}}$, where $p_{i}$ and $p_{j}$ are the pressure in pores $i$ and $j$, and $L_{i j}$ the distance between the pore centers. The local conductance $g_{i j}$ depends on the geometry of the fluid domain on the face connecting pores $i$ and $j$ and the fluid viscosity. Greater detail on this scheme can be found in Chareyre et al. [22] together with a validation against finite element simulations.

In the PFV approach, the fluid problem is thus reduced to the inversion of a sparse matrix to obtain a piecewise constant pressure field. As a result, this approach makes it possible to tackle fluid grain interaction problems in 3D. By integrating pressure and viscous stresses on each grain, the impact of the internal flow is taken into account in the DEM computation by adding fluid forces on each grain of the simulation [22].

\section{Sample generation}

A cubic assembly of spheres is generated randomly with a uniform radius distribution between $r_{\min }$ and $r_{\max }=3.5 r_{\min }$ and compacted using the radius expansion technique up to $20 \mathrm{kPa}$. During this process, the inter-particle friction angle is maintained at its value of $35^{\circ}$ to prepare a loose granular material with a $58 \%$ pack- 


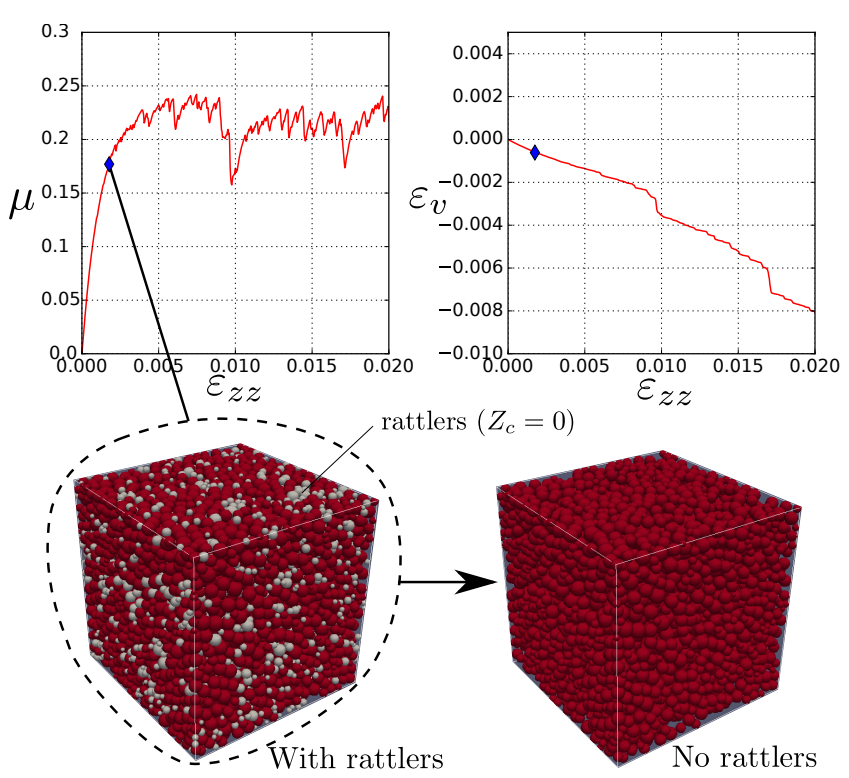

FIG. 2. Drained triaxial test response with respect to axial strain $\varepsilon_{z z}$ (top). The Drucker-Prager stress ratio $\mu$ is defined as the ratio between the maximum shear stress and the mean stress, and $\varepsilon_{v}$ stands for the relative volume change. The diamond corresponds to the mechanical state $\mu=0.175$ considered for the fluid/grain coupled problem. The two bottom images illustrate the microstructure changes resulting from the removal of rattlers (particles with coordination number $\left.Z_{c}=0\right)$. The 3,458 particles removed are shown in white.

ing fraction. At the scale of the whole sample, macroscopic diagonal stress and strain tensors are defined from boundary forces and displacement, and are denoted hereafter as $\boldsymbol{\sigma}$ and $\varepsilon$, respectively, with non-zero components $\sigma_{i i}$ and $\varepsilon_{i i}, i \in\{x, y, z\}$.

From the equilibrium stateobtained, a drained triaxial loading is then imposed on the sample under a lateral confining stress $\sigma_{x x}=\sigma_{y y}=100 \mathrm{kPa}$ and a vertical compression strain rate $\dot{\varepsilon}_{z z}=-0.01 \mathrm{~s}^{-1}$. The mechanical and volumetric responses are shown in Figure 2 with respect to the vertical strain $\left|\varepsilon_{z z}\right|$. The behavior observed is typical of non-compacted granular materials as:

- the Drucker-Prager stress ratio $\mu$ increases as the vertical compaction increases. By definition, $\mu$ is the ratio between the maximum shear stress $\tau$ and the mean pressure $p$. In axisymmetric conditions $\tau=\frac{\sigma_{z z}-\sigma_{x x}}{2}$ and $p=\frac{1}{3}\left(\sigma_{z z}+2 \sigma_{x x}\right)$ as $\boldsymbol{\sigma}$ is diagonal with $\sigma_{x x}=\sigma_{y y}$.

- the relative volume change $\varepsilon_{v}$ monotonously decreases as the vertical compaction increases.

During this triaxial loading, one sample is saved for $\mu=0.175$ (this state is marked with a diamond in Figure 2) and deprived of rattlers as illustrated in Figure 2. As shown in Wautier et al. [33], the resulting sample is known to be mechanically unstable in the sense of the second order work criterion. Some incremental loading programs will result in an unbounded increase in kinetic energy, which is a signature of an inertial transition. However, knowing whether additional forces resulting from an internal fluid flow can trigger this inertial transition remains an open question.

\section{Numerical testing}

The numerical sample resulting from the preparation procedure presented in the previous subsection is then subjected to a macroscopic pressure gradient. During this numerical testing, the stress state is kept constant $(\mu=0.175)$ and the microstructure is left free to evolve (in particular, new rattlers are not removed). Two flow directions are considered:

- parallel to the principal loading direction (along $z$ axis) and referred to as the 'vertical flow case' hereafter;

- perpendicular to the principal loading direction (along $x$ axis) and referred to as the 'horizontal flow case' hereafter.

Figure 3 summarizes the boundary value problem solved in the two flow cases. On the upstream faces, the fluid pressure is set at $p_{0}$ while on the downstream faces null fluid pressure and null displacement are imposed. On the remaining faces of the sample, zero flux conditions are imposed $\left(q_{x}=q_{y}=0\right.$ for the vertical case and $q_{z}=q_{y}=0$ for the horizontal case). The upstream pressure $p_{0}$ is prescribed such that the resulting pressure gradients correspond to the usual values observed in natural granular materials subjected to internal flows. In the vertical case, $p_{0}=151 \mathrm{~Pa}$ while in the horizontal case $p_{0}=152 \mathrm{~Pa}$.

In Figure 4, the incremental strain responses are shown for the two flow directions while the initial and final microstructures are shown in Figure 5 (see supplementary material at [url will be inserted by publisher] for videos corresponding to the two flow cases). In both cases, the collapse of the sample is observed with very large final incremental strains in the principal direction of loading whatever the flow direction $\left(\left|\mathrm{d} \varepsilon_{z z}\right| \simeq 12-14 \%\right)$. The failure direction is indeed governed by the stress state. In the present case $(\mu>0)$, the compression in the direction $z$ is greater than the transverse compression in the directions $x$ and $y$. When material failure occurs, the sample undergoes a transient liquefaction during which it can no longer withstand the imposed mechanical loading [34]. During this transient period, it shrinks along the direction of major compression ( $z$ in the present case). The deformation stops when the new microstructure was able to resist the imposed stress state again. The collapse direction could switch provided that the additional fluid pressure $p_{0}$ imposed in the horizontal direction was such that $p_{0}+\sigma_{x x}>\sigma_{z z}$. This is unlikely to occur, however, because in practice $p_{0} \simeq 10^{2} \mathrm{~Pa} \ll \sigma_{x x}=\sigma_{y y} \simeq 10^{5} \mathrm{~Pa}$.

Indeed, the internal flow acts as a small perturbation that is sufficient to trigger the collapse, but it does not 


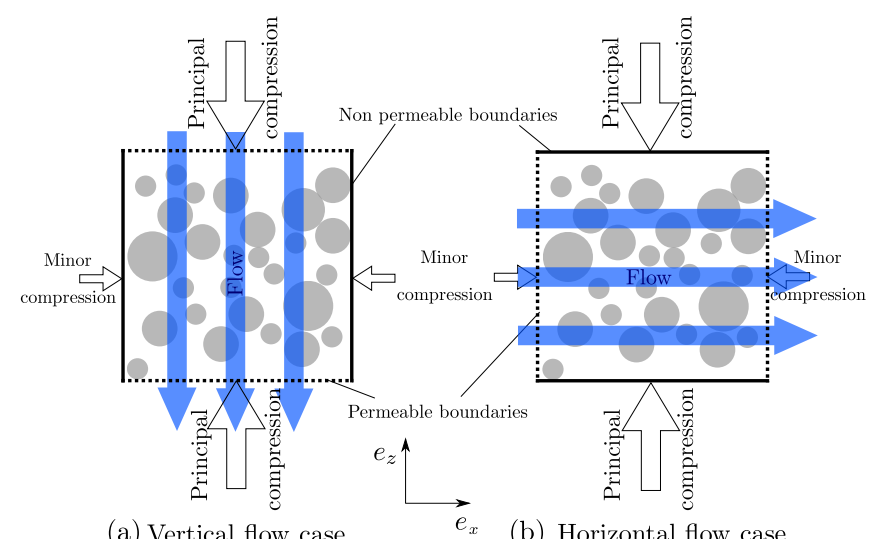

(a) Vertical flow case

(b) Horizontal flow case

FIG. 3. Boundary value problems solved for two flow directions. In the vertical case (a), zero flux boundary conditions are applied in directions $x$ and $y$ while on the top face a pressure $p_{0}$ is imposed while keeping $p=0$ on the bottom face. In the horizontal case (b), zero flux boundary conditions are applied in directions $z$ and $y$ while on the left face a pressure $p_{0}$ is imposed while keeping $p=0$ on the right face. In both cases, the mechanical loading corresponds to the stress state $\mu=0.175$ reached during the triaxial loading of Figure 2. In the $x$ and $y$ directions a confining stress $\sigma_{x x}=\sigma_{y y}=100 \mathrm{kPa}$ is imposed while in the $z$ direction a compression $\sigma_{z z}=\frac{4 \mu+1}{1-2 \mu} \sigma_{x x}$ is applied.

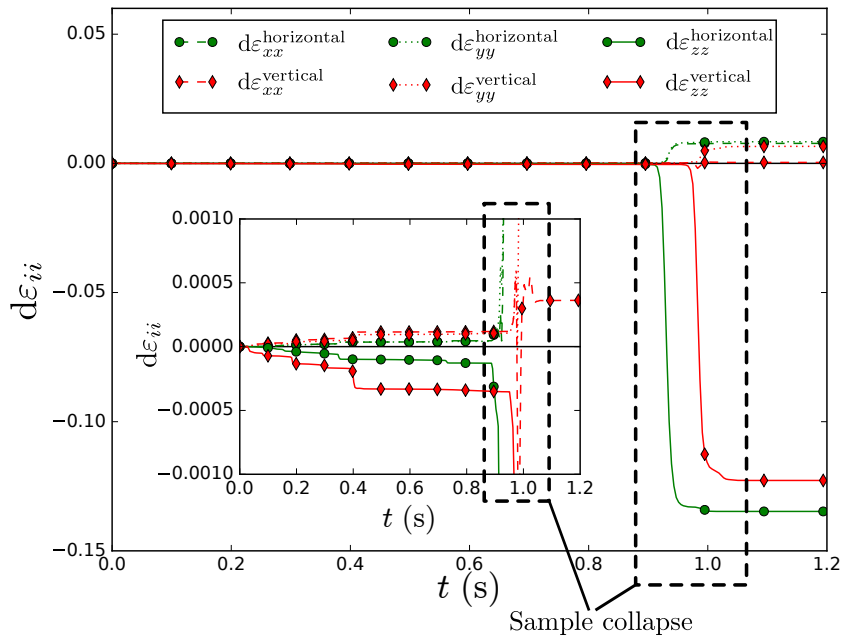

FIG. 4. Incremental strain response with respect to time after a fluid pressure drop $p_{0}$ is applied in the vertical direction (red curves) or in the horizontal direction (green curves) while keeping a stress state constant $(\mu=0.175)$.

control the failure direction. In addition, the flow direction does not seem to have any influence on the occurrence of failure.

In Figure 4, the collapse of the sample is visible as $\mathrm{d} \varepsilon_{z z}$ suddenly drops below -0.1 , which corresponds to a greater than $10 \%$ decrease of the sample length in the principal stress direction (see Figure 5). Laterally, a slight dilation of roughly $1 \%$ is observed in the mean-

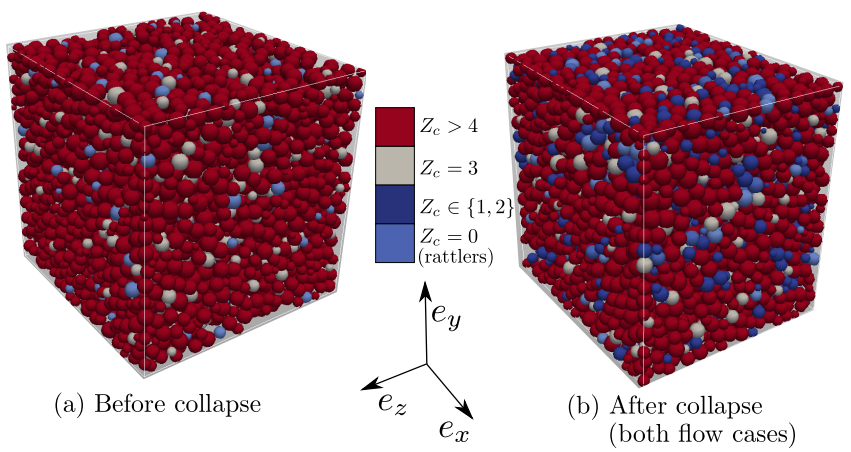

FIG. 5. Sample visualization before and after fluid flow is applied. Particles are colored according to their coordination number $Z_{c}$ (see supplementary material at [url will be inserted by publisher] for videos corresponding to the two flow cases).

time. In the inset plot of Figure 4 small strain jumps corresponding to small microstructure changes are observed before the global collapse. Indeed, the fluid impact on the granular skeleton is incremental and the flow-induced modifications of the contact network develop with a characteristic time up to the overall failure.

\section{MICROMECHANICAL INSPECTION OF THE SAMPLE COLLAPSE}

In this Section we investigate the micro-origin of the global collapse observed after a fluid flow is created in the granular sample considered in the previous section in which rattlers were removed initially.

\section{A. Fluid force fluctuations}

In the boundary value problems summarized in Figure 3, the confining stresses applied through grain contacts by the bounding walls are kept unchanged while a fluid pressure drop is imposed to generate an internal fluid flow. As reviewed in Section II B, the flow impact is then taken into account in the DEM computation through additional fluid forces applied on every particle. As a result, these additional fluid forces are at the origin of the sample collapse. In Figure 6, their micro-influence is assessed by plotting the statistical distributions of their three components $F_{x}^{f}, F_{y}^{f}$ and $F_{z}^{f}$ for the two flow cases at the beginning of the flow simulation $(t=0 \mathrm{~s})$. The probability density functions (pdf) shown in Figure 6 remain unchanged until the global failure of the sample (not shown here).

In Figure 6 only two functions are sufficient to describe the six pdfs:

- one to account for fluid forces along the direction of the macroscopic pressure gradient $(x$ or $z)$. These pdfs have strictly positive mode and mean values, 


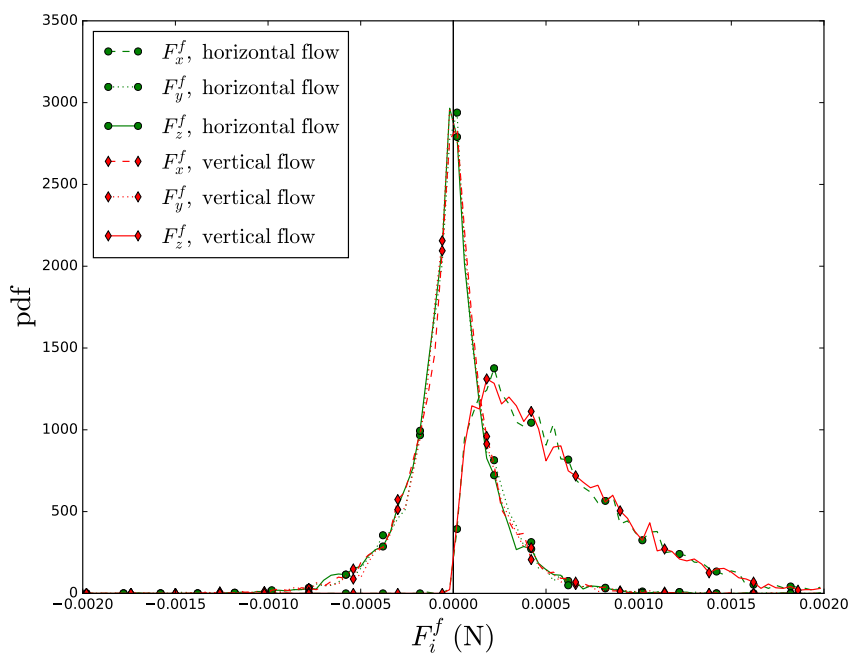

FIG. 6. Probability density functions (pdfs) associated with fluid force components $F_{x}^{f}, F_{y}^{f}$ and $F_{z}^{f}$ acting on individual grains after a fluid pressure drop $p_{0}$ is applied in the vertical direction (red curves) or in the horizontal direction (green curves) while keeping a stress state constant $(\mu=0.175)$. The pdfs are plotted for $t=0 \mathrm{~s}$ but remain unchanged until the global failure of the sample.

which is consistent with the macroscopic flow direction.

- one to account for fluid forces perpendicular to the macroscopic pressure gradient ( $y$ and $z$ or $x$ and $y$ ). These pdfs are symmetric with zero mean values, which is also consistent with the macroscopic flow direction.

As a result, the pore network driving the fluid force distributions remains isotropic even if the stress state is anisotropic. This is indeed due to the fact that the axial strain levels reached during the triaxial loading are too small to lead to microstructure anisotropy [18].

The similarities between the pdfs in Figure 6 and those measured experimentally $[20,35,36]$ or numerically using other coupling schemes [37, 38] are striking. In case of Stoke drag force, the fluid force is proportional to the flow velocity. As a result, a comparison between velocity and force pdfs may be attempted by rescaling the fluid forces by the mean fluid force in the macroscopic flow direction. The comparison between pdfs from Figure 6 and those obtained by Beguin et al. [20] is shown in Figure 7.

The pdfs for the direction perpendicular to the macroscopic flow are very close to each other while a greater difference can be observed in the macroscopic flow direction. In particular, the experimental mode is much smaller than the mode obtained numerically. This can be explained because the pdfs obtained in Beguin et al. [20] are computed from velocity fields at a resolution finer than the pore size. Indeed, Beguin's statistical analysis [20] accounts for the variations of the fluid velocity in

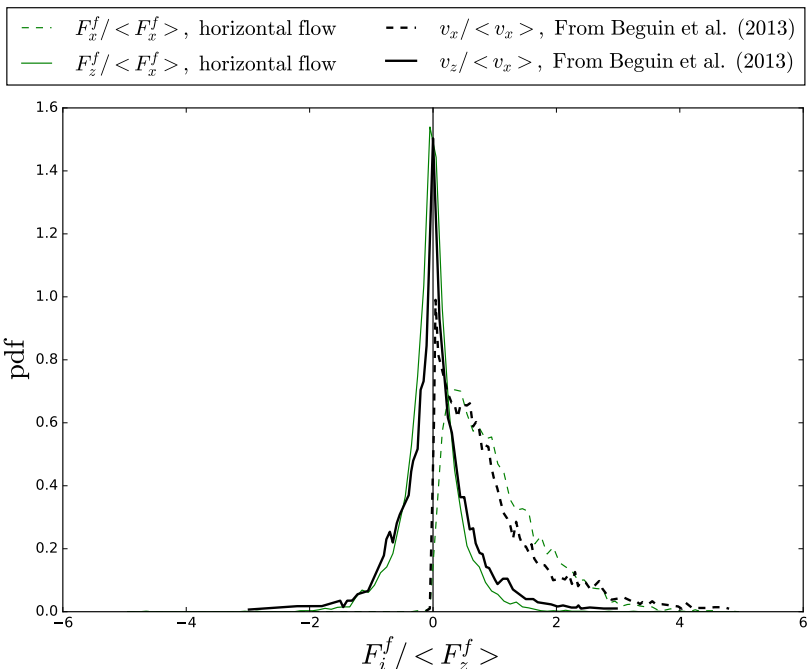

FIG. 7. Probability density functions (pdfs) associated with normalized fluid forces $F_{x}^{f} /<F_{x}^{f}>$ and $F_{z}^{f} /<F_{x}^{f}>$ in the horizontal direction (green lines) compared to the pdfs obtained in Beguin et al. [20] for dimensionless fluid velocities $\left.v_{x}^{f} /<v_{x}^{f}\right\rangle$ and $\left.v_{z}^{f} /<v_{x}^{f}\right\rangle$ (thick black lines).

boundary layers around grains. As a result, greater contribution of low velocity values is expected as the fluid velocity is nil on the grain boundaries.

The comparison between the two functions describing the pdfs in Figure 6 also gives important clues to understand why the fluid flow direction does not influence the occurrence of macroscopic failure. Even if fluid forces perpendicular to the macroscopic pressure gradient have a zero mean value, the pdf shows a standard deviation that is non-negligible compared to the pdf mode corresponding to the component along the macroscopic flow direction. As a result, whatever the macroscopic flow direction, local fluid force fluctuations can result in incremental loading to particles such that some of the current contacts will reach the Mohr-Coulomb limit sliding condition. This situation is illustrated in Figure 8. From a quantitative point of view, imagine for instance that a given particle contact is about to slide provided that an additional fluid force $F_{z}>0.0002 \mathrm{~N}$ is applied. Based on the statistical results shown in Figure 6, the probability of this contact sliding is $80 \%$ in the vertical flow case and $15 \%$ in the horizontal one. As a result, even for the horizontal flow case, this contact has a nonnegligible chance sliding. The local non-sensitivity to the macroscopic flow direction is only true, however, for lowmagnitude forces. Large fluid forces are more aligned to the macroscopic flow direction, as shown by the pdfs in Figure 6. The ability of both flow directions to trigger the sample's collapse means that the required magnitude of the fluid forces is quite small for the microstructure considered and the stress state considered (at least on the same order of magnitude as the mode value of the pdf corresponding to the macroscopic flow direction). 


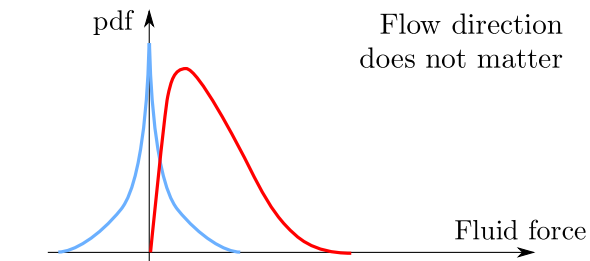

Non negligible force fluctuations in direction perpendicular to the macroscopic flow direction

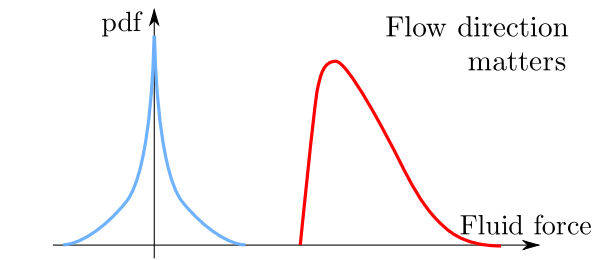

Negligible force fluctuations in direction perpendicular to the macroscopic flow direction

FIG. 8. Two possible cases for the fluid force pdfs in the direction of the macroscopic flow and perpendicular to it. In the top case (observed situation), pdfs overlapping means that force fluctuations cannot be neglected. In the bottom case (hypothetic situation), no overlapping exists and force fluctuations around the macroscopic flow direction can be ignored.

\section{B. From local to global failure}

For a material in the bifurcation domain, some incremental loadings will result in an unbounded increase in kinetic energy. As a result, it is of particular interest to have a close look at the grain's kinetic energy to analyze the overall failure at the microscale. The local changes in the kinetic energy are shown in Figure ?? and ?? in which particles are colored according to their kinetic energy, and the most energetic ones are highlighted using an arbitrary threshold $E_{c}^{*}$. Videos corresponding to Figure ?? and ?? are available in the supplementary material available at [url will be inserted by publisher].

For both flow directions the sample collapse is preceded by a double burst of kinetic energy. The two bursts initiate locally and then propagate to the whole sample in quite a similar pattern, as shown in Wautier et al. [34] in the case of an incremental stress loading associated with a negative second-order work. If the first bursts initiate in different locations for the two flow directions, the second bursts start at the same spot. For the vertical flow case the two bursts follow in a row while for the horizontal flow case, the sample temporarily restabilizes between the two outbursts in kinetic energy. As a result, the second burst is more intense in the vertical case and a higher kinetic threshold $E_{c}^{*}=10^{-6} \mathrm{~J}$ was used to highlight its propagation.

The burst mechanism is a signature of a local failure of the existing contact network under the action of additional fluid forces. Even if the failure remains localized, this can affect the whole contact network as stresses are forced to redistribute. To assess the impact of this stress redistribution on the contact network, it is interesting to analyze whether contact forces get closer to the MohrCoulomb limit. At the contact scale a sliding index may be defined as:

$$
I_{p}=\frac{\left\|\boldsymbol{F}_{t}\right\| /\left\|\boldsymbol{F}_{n}\right\|}{\tan \phi} \in[0,1]
$$

where $\phi$ is the inter-particle friction angle, $\boldsymbol{F}_{t}$ and $\boldsymbol{F}_{n}$ are respectively the tangential and normal contact forces as defined in Figure 1.

$I_{p}$ values sufficiently close to 1 is a necessary condition to observe the vanishing of the second-order work locally $[21,39]$. A large population of contacts with $I_{p}$ close to 1 is therefore a necessary condition to observe material instability, i.e., macroscopic vanishing of the second-order work [34]. In Figure 10, the fraction of contacts close to sliding $\frac{N_{c}\left(I_{p}>0.9\right)}{N_{c}^{\text {tot }}}$ is plotted over time for the two flow cases during the sample collapse identified in Figure 4 by large strain jumps. In this figure, the initiation of the two outbursts of kinetic energy detected in Figure ?? and ?? are shown with vertical solid lines.

In Figure 10, the two bursts of kinetic energy identified have two very different signatures. While the first burst is associated with a decrease in $\frac{N_{c}\left(I_{p}>0.9\right)}{N_{c}^{\text {tot }}}$, the second burst is associated with an increase in $\frac{N_{c}\left(I_{p}>0.9\right)}{N_{c}^{\text {tot }}}$. As the first outbursts in kinetic energy result in building more resilient contact networks with fewer contacts likely to reach the Mohr-Coulomb condition $\left(I_{p}=1\right)$, the second bursts result in less resilient contact networks, which eventually fail globally.

\section{Mesoscale mechanisms}

As widely acknowledged in the literature, stresses imposed at the macroscopic scale concentrate locally on only $20-30 \%$ of the grains organized in elongated mesostructures commonly called force chains [25-27, 30]. The interplay between these chains and the surrounding loosely stressed particles (the non-chained grains) is known to have important implications on force chain stability because non-chained particles provide lateral support $[29,40]$. Using the force chain definition introduced by Peters et al. [28], the set of chained particles can be tracked in the sample considered during its flow-induced collapse identified in Figure 5.

In Figure 11, the current set of chained particles is compared to the initial one in terms of:

- born particles $\left(N_{\text {born }}\right)$ : current chained particles that did not belong to the initial force chains;

- dead particles $\left(N_{\text {died }}\right)$ : initially chained particles that no longer belong to force chains.

In Figure 11, the sample collapse identified in Figure 4 by large strain jumps is visible through significant modifications in the chained particle populations (after collapse, roughly $70 \%$ of the initial chained particles have 'died'). 


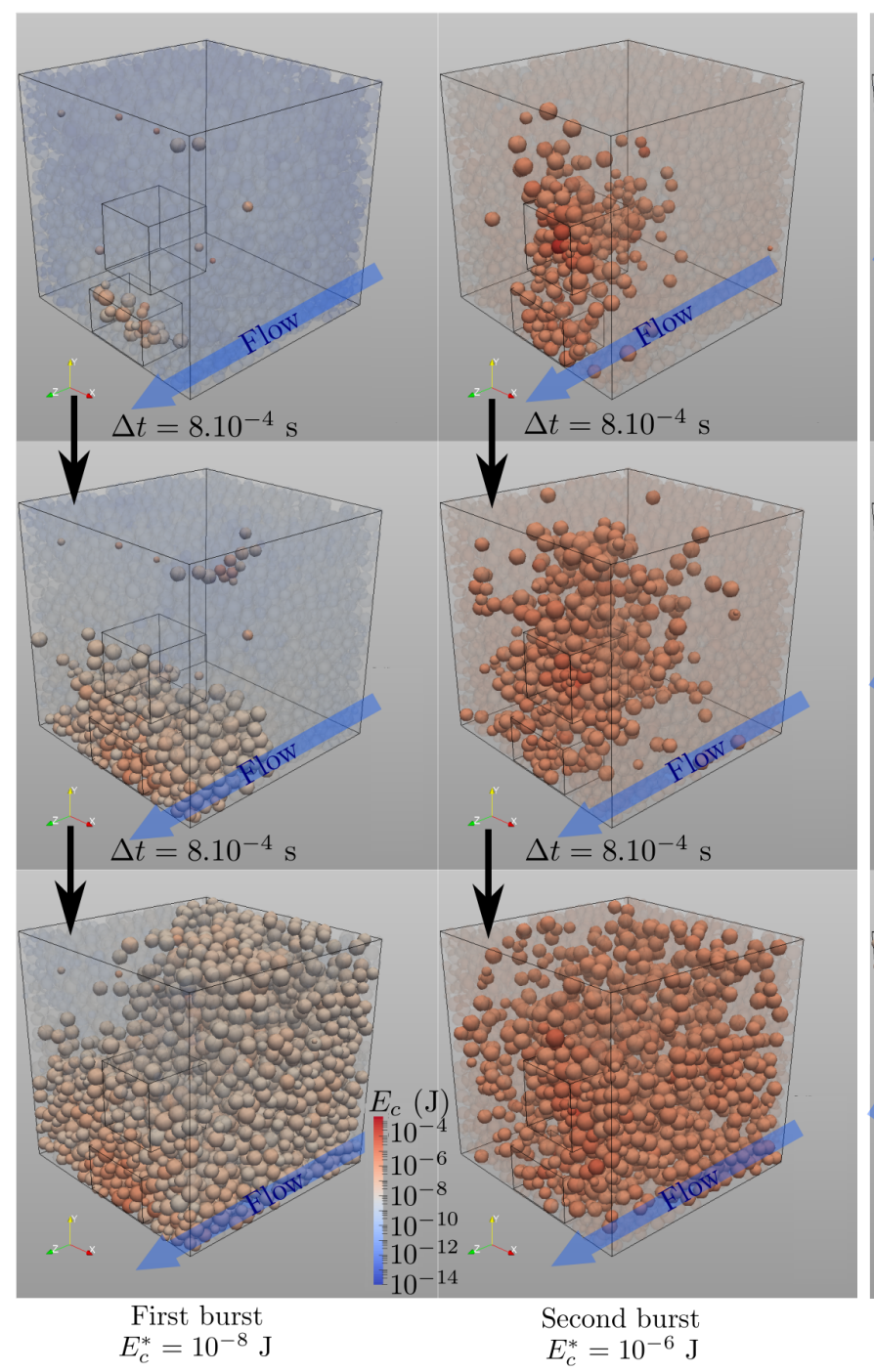

(a) Vertical flow case

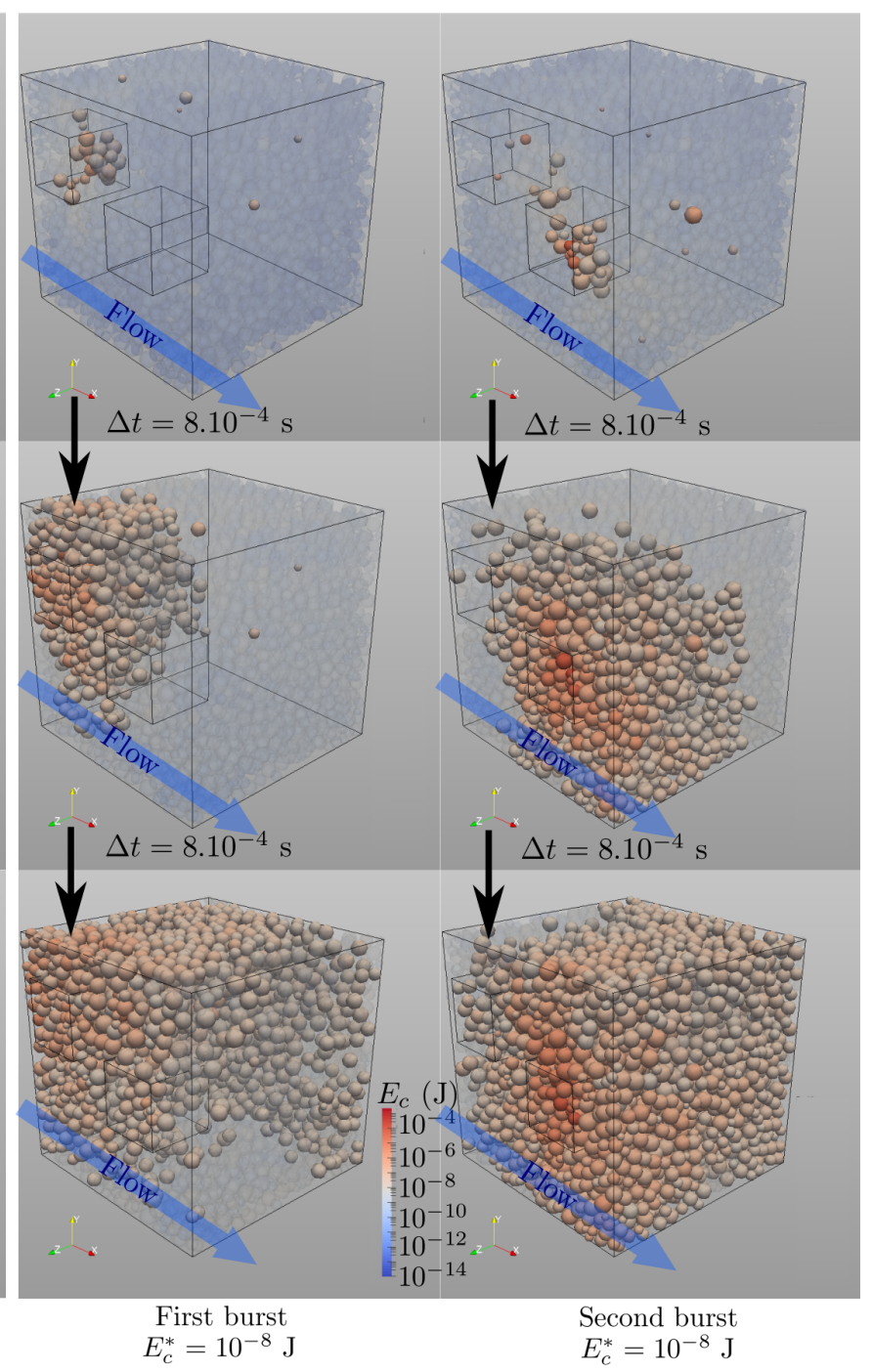

(b) Horizontal flow case

FIG. 9. Visualization of the two outbursts in kinetic energy propagation for the two flow cases. Particles are colored according to their kinetic energy (log scale). Those with kinetic energy higher than $E_{c}^{*}$ are highlighted.

A simultaneous increase in $N_{\text {born }}$ and $N_{\text {died }}$ occurs as outbursts in kinetic energy are observed. In the end, as the sample gets denser, more force chains are built than initially $\left(N_{\text {born }}>N_{\text {died }}\right)$.

Elementary force chain bricks are composed of three particles. They are referred to as ' 3 -p groups' in the following. At some point, they may not be identified as such because they no longer satisfy one of the three conditions used in the force chain definition [28] and illustrated in Figure 12:

- a contact is lost between the three particles considered.

- one of the particle's principal stresses is now lower than the mean principal stress computed for all the sample particles.

- the geometrical direction of the contacts is no longer aligned with the principal stress direction (a maximum deviation of $45^{\circ}$ is used here).

In Figure 13 the relative contributions of the above causes for the disappearance of a 3-p group are given for the current set of 3-p groups that will disappear in the future.

Misalignment between principal stress and contact directions and relative unloading compared to the mean principal stress are the two main causes of the disappearance of initial 3-p groups. In contrast, contact loss is not a significant mechanism at first. As a result, the changes in force chain populations observed in Figure 11 mainly result from force chain bending occurring simultaneously with the second outburst in kinetic energy.

Once the general collapse occurs (after the second outburst in kinetic energy), the remaining force chains will mainly disappear because of stress redistribution (rel- 
(a) Vertical case

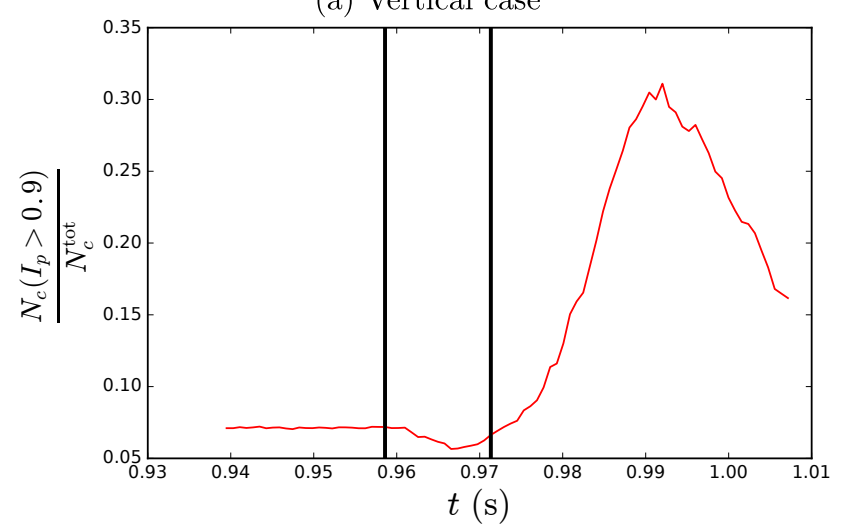

(b) Horizontal case

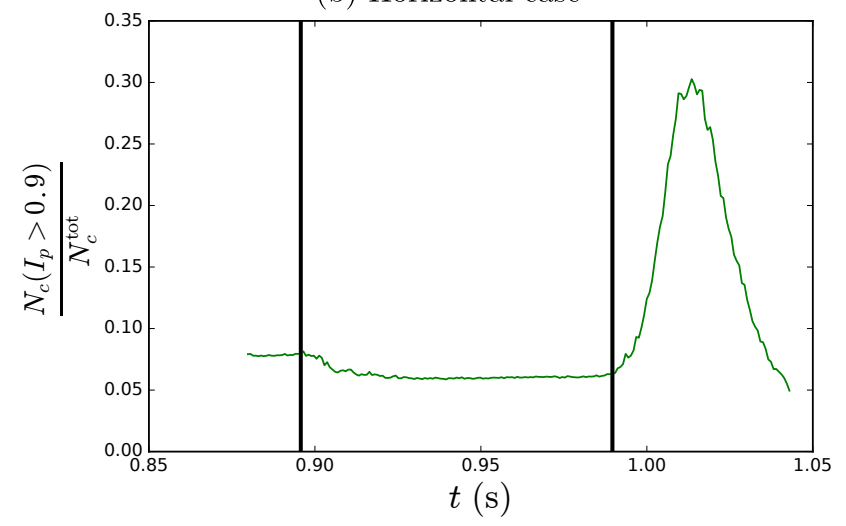

FIG. 10. The fraction of contacts close to sliding over time during the sample collapse for the vertical flow case (a) and the horizontal case (b). The onset of the two outbursts in kinetic energy is shown with vertical solid lines.

ative unloading compared to the mean principal stress value). Later on, contact loss becomes a non-negligible cause for 3-p groups' disappearance. These observations are consistent with previous results found in the literature for dry granular materials describing the micromechanisms responsible for failure in granular materials $[29,34,40]$. Additional fluid forces induce force chain bending, which results in considerable stress redistribution and eventually some contact losses. It is important to emphasize here that the disappearance of 3-p groups through contact loss is a consequence of the global collapse of the contact network and not directly driven by the fluid flow. As a result, grain detachment is not directly induced by fluid flows but is a consequence of force chain collapse.

Based on the distinction between chained particles (heavily stressed particles constituting the force chains) and non-chained particles (loosely stressed particles not belonging to force chains), three populations of contacts should be defined, namely chained/chained $(c c)$ nonchained/non-chained $(n n)$ and non-chained/chained $(n c)$ contacts. By tracking how these three contact populations evolve over time, information can be obtained on (a) Vertical case

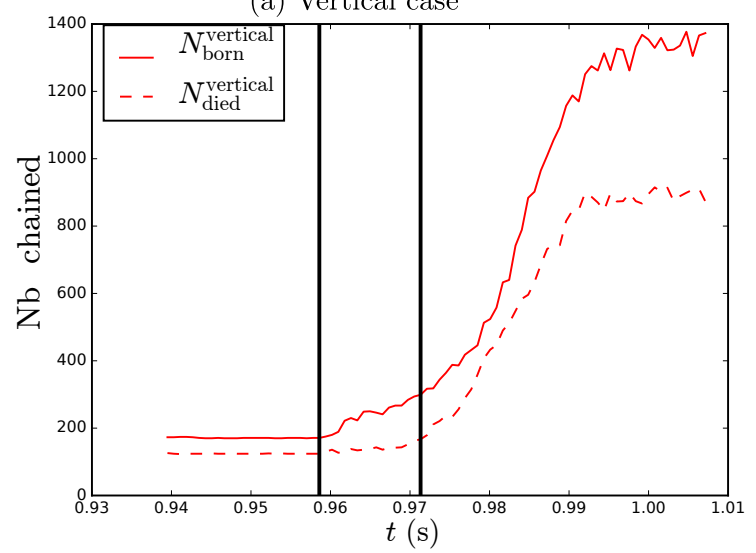

(b) Horizontal case

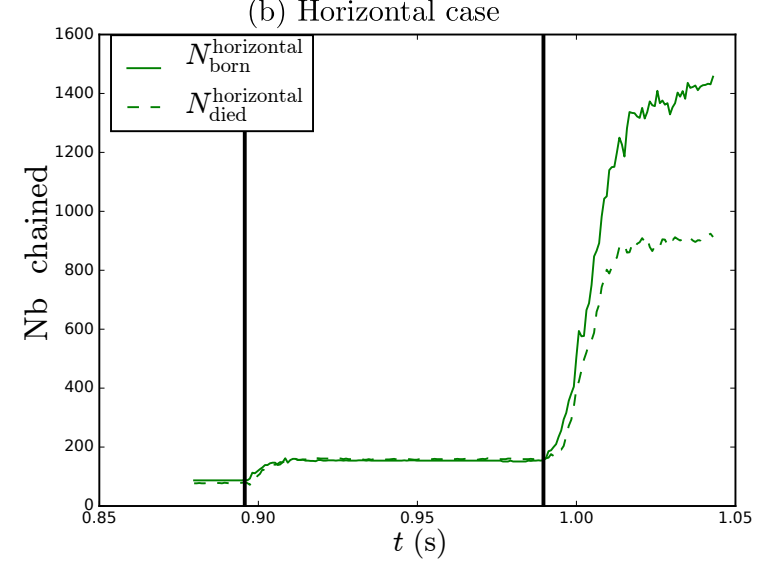

FIG. 11. $N_{\text {born }}$ (solid) and $N_{\text {died }}$ (dashed) particles during the sample collapse for the vertical flow case (a) and the horizontal case (b). The common reference set of chained particles is defined on the sample prior to imposing the horizontal or vertical fluid pressure drop. The onset of the two outbursts in kinetic energy is shown with vertical solid lines.

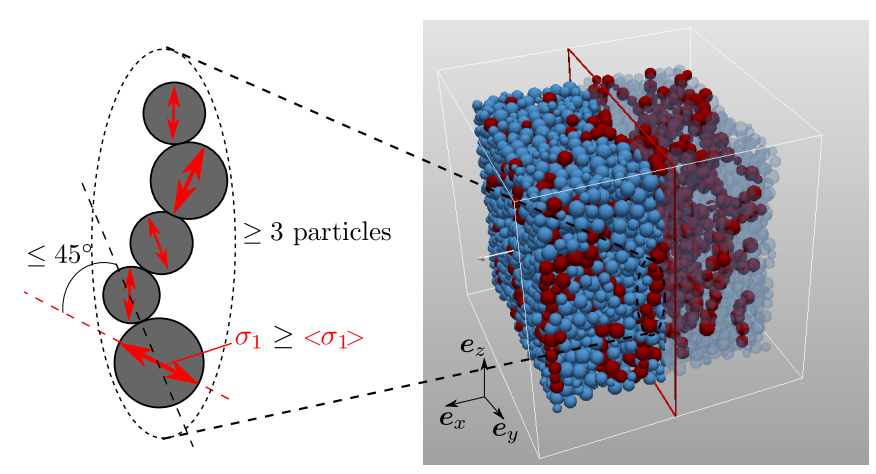

FIG. 12. Three-criteria force chain definitions according to Peters et al. [28] (left) and force chain visualization in the sample before collapse (right). $\sigma_{1}$ corresponds to the principal compression value of each grain. Grains belonging to force chains are colored in red while non-chained grains are represented in blue or light blue. 


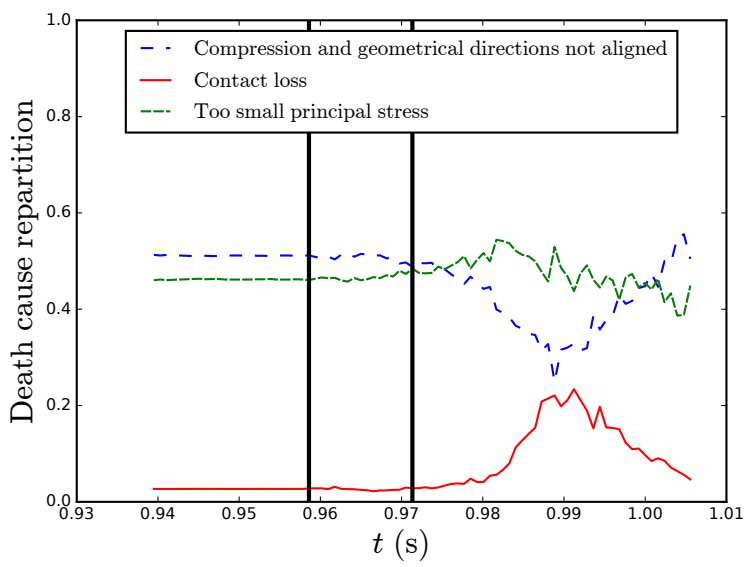

(a) Vertical case

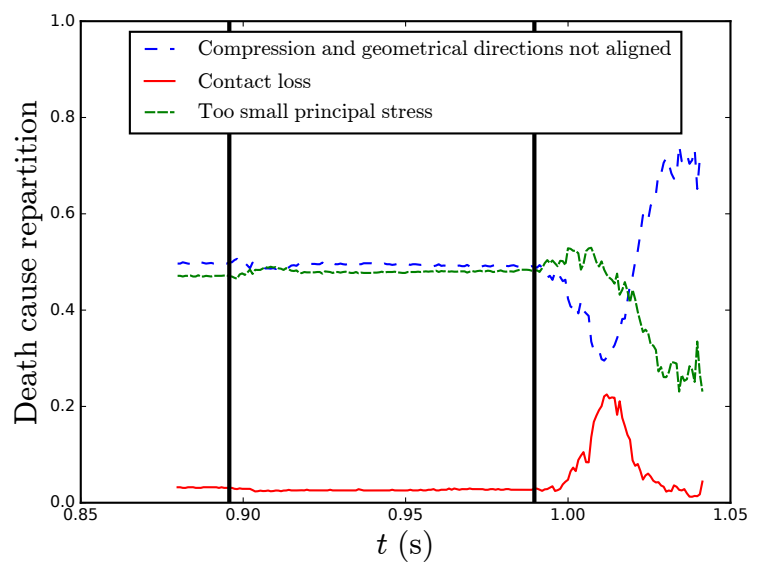

(b) Horizontal case

FIG. 13. Statistical distribution between the three possible causes of disappearance for 3-p groups existing at time $t$ and disappearing at time $t_{f}<1.01 \mathrm{~s}$ (vertical flow case (a)) or $t_{f}<1.05 \mathrm{~s}$ (horizontal case (b)). A 3-p group may no longer be identified as such, either because of contact loss (solid line), relative unloading (densely dashed line) or geometrical/compression misalignment (dashed line). The onset of the two outbursts in kinetic energy is shown with vertical solid lines.

internal kinematic constraints. In particular, the number of non-chained/chained contacts $\left(N_{n c}\right)$ is known to be of fundamental importance with respect to force chain stability $[34,40]$. In Figure $14(\mathrm{a})$ and $14(\mathrm{~b}), N_{n c}, N_{n n}$ and $N_{c c}$ evolving over time is shown for three control volumes: for the whole sample and for two small volumes around the locations of the two outbursts in kinetic energy. The control volumes located around the positions of the outbursts in kinetic energy are visible in Figure ?? and ??.

For the horizontal case in Figure 14(b), the first outburst in kinetic energy is followed by a local drop in $N_{n n}$ together with an increase in $N_{n c}$ and $N_{c c}$. As a result kinematic constraints increase around force chains, which is consistent with the sample recovering from temporary destabilization. This is not the case for the second burst, accompanied by a drop in $N_{n c}$, which characterizes force chain deconfinement. This drop first appears locally in the second control volume $\left(N_{n c}^{b_{2}}\right)$ before generalizing to the whole sample $\left(N_{n c}\right)$. In the end, as the sample densifies and new force chains are built, $N_{n n}, N_{c c}$ and $N_{n c}$ increase again.

For the vertical case in Figure 14(a), the two bursts appear in a row with increasing levels of kinetic energy. Although the first burst is not accompanied by a drop in $N_{n c}$, the second burst is. As for the horizontal case, the drop in $N_{n c}$ first appears locally in the second control volume $\left(N_{n c}^{b_{2}}\right)$ before generalizing to the whole sample $\left(N_{n c}\right)$. As the sample densifies $N_{n n}, N_{c c}$ and $N_{n c}$ increase again in the end.

In both cases, the sample collapse occurs simultaneously with a release in kinematic constraints around force chains, which is consistent with previous results found in the literature [34, 40]. However, it must be emphasized that this force chain deconfinement does not seem to be the triggering mechanism, as was shown in the case of an incremental stress loading corresponding to a nega- tive second-order work [34]. Indeed, for classical stresscontrolled directional analysis under axisymmetric conditions, the vanishing of the second-order work is usually observed for loading directions corresponding to a macroscopic dilation $[13,34,41]$. At the microscale, this dilation results in some contact loss. When occurring around force chains, contact loss results in a decrease in kinematic constraints, which eventually lead to force chain collapse. In the present case, the perturbation is imposed by the fluid and does not necessarily result in global dilation. As a result, no force chain deconfinement is observed before the onset of the outburst in kinetic energy in Figure 14.

\section{CONCLUSION}

With the initial removal of rattlers, the direct impact of a fluid flow on the primary fabric of a granular material was investigated in terms of mechanical stability. For a reference state (stress and microstructure geometry) identified as mechanically unstable, it was found that:

- A fluid flow is able to trigger the underlying instability on its own (without any change in the external contact forces on the sample boundaries).

- The triggering is not instantaneous and before the final collapse small reorganizations are observed together with drops in the number of contacts close to sliding and limited incremental strain jumps.

- Because of fluid force fluctuations in the directions perpendicular to the macroscopic flow, the flow direction does not have any influence on the triggering of the underlying instability.

- The sudden collapse results from an outburst in kinetic energy mechanism associated with force chain 

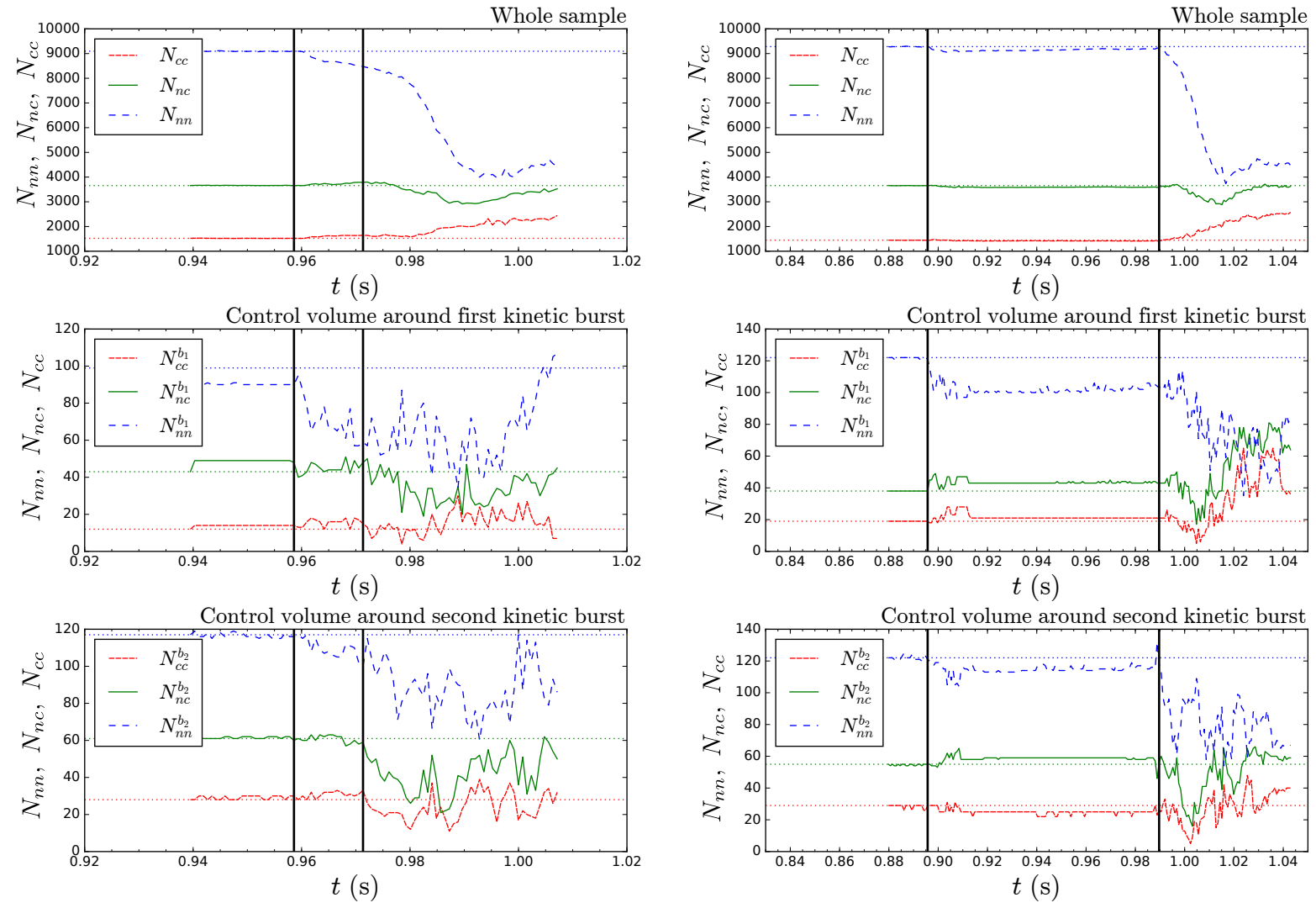

(a) Vertical flow case

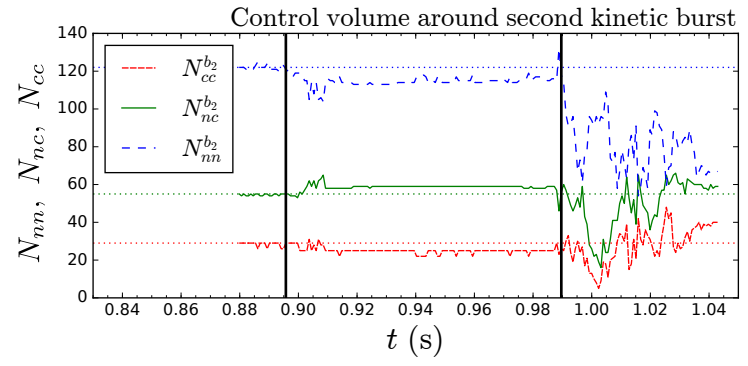

(b) Horizontal flow case

FIG. 14. The number of chained/chained (densely dashed line) non-chained/non-chained (dashed line) and non-chained/chained (solid line) contacts during the sample collapse for the two flow cases. $N_{n c}, N_{n n}$ and $N_{c c}$ are given for the whole sample and for the two control volumes shown in Figure 9. The onset of the two outbursts in kinetic energy is shown with vertical solid lines.

deconfinement and an increase in the number of contacts close to sliding.

Note that while we remove rattlers from the sample, they still play an important role in the mechanical stability of granular materials. By getting jammed when existing contact networks fail, rattlers prevent the development of large irreversible deformations that contribute to ensuring mechanical stability [21]. Rattlers are also highly sensitive to fluid flow and they may be either eroded or clogged. By looking at the unmodified microstructure, it will be possible to assess whether erosion and clogging could mitigate the findings of this paper in terms of flow-triggered instabilities.

\section{Appendix A: Benchmark results without initial rattler removal}

The initial removal of rattlers creates contrived microstructures that are not representative of real granular materials. This procedure made it possible to isolate the direct impact of a fluid on the primary skeleton of gran- ular materials, and this was the main goal of this study. However, to open the door to the study of more realistic microstructures, in this appendix we analyze the results obtained for the vertical flow case illustrated in Figure 3 while keeping the initial rattlers in the sample. A short video of this numerical simulation is available in the supplementary material at [url will be inserted by publisher]. In this configuration, the fluid is unable to trigger the sample's collapse. The reasons for this non-collapse can be analyzed by comparing the spatial distribution of the fluid pressure with and without rattlers. In Figure 15, the pore pressures are plotted against their position in the macroscopic flow direction ( $z$ in the vertical flow case).

Figure 15 shows that in both cases, the mean pore pressure follows the macroscopic pressure gradient with some fluctuations. When rattlers are removed, the pressure fluctuations increase, which results in larger fluctuations in the fluid forces, as shown in Figure 16.

As a result, the perturbation introduced by the fluid flow is greater when rattlers are absent, which her results in the triggering of the underlying material's instability. By removing rattlers before applying a fluid pressure drop, 


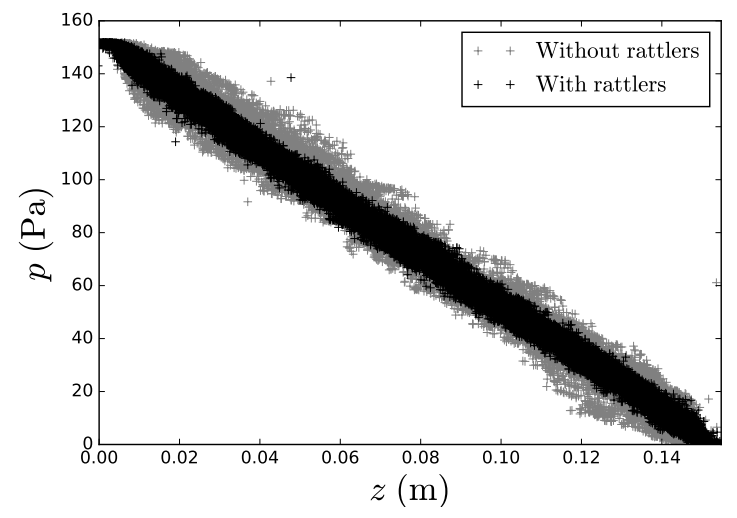

FIG. 15. Pore pressures in the macroscopic flow direction just after a macroscopic fluid pressure drop is applied in direction $z$. Black dots correspond to the case for which rattlers are kept in the sample, while gray dots correspond to the case for which rattlers are removed from the sample. In both cases the stress ratio $\mu=0.175$ is kept unchanged while applying the fluid pressure drop.

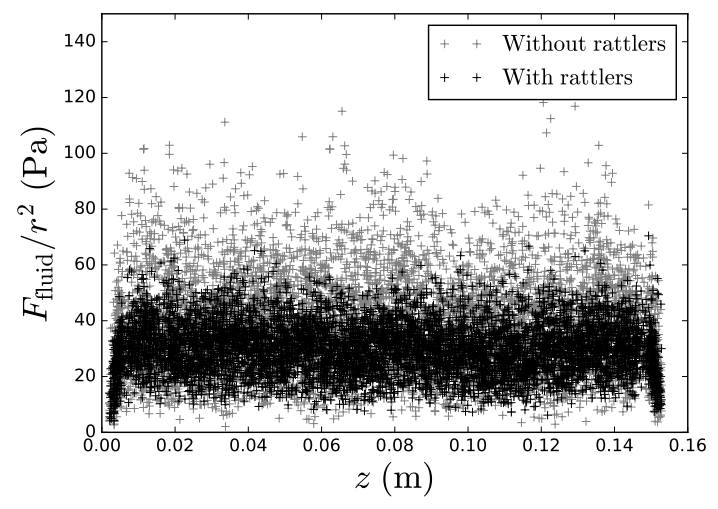

FIG. 16. Fluid forces divided by the square of the grain radii just after a pressure drop is applied in the $z$ direction. Only non-free particles are considered in the plot. Black dots correspond to the case for which rattlers are kept in the sample, while gray dots correspond to the case for which rattlers are removed from the sample.

the idea developed in this paper was to isolate the direct impact of the fluid on the primary skeleton to highlight the importance of the local pressure fluctuations in the triggering of material instability. The results shown in this appendix underline once again the importance of local fluid force fluctuations in the triggering of material instability. This finding also justifies a posteriori the choice to solve the fluid problem locally at the pore scale and not directly at the macroscopic scale using Darcy's law and the Kozeny-Carman permeability expression, for instance. In such unresolved approaches, fluid forces are always parallel to the macroscopic direction of the fluid flow and no fluctuations are captured despite their importance in the occurrence of material failure.
[1] S. Bonelli, Erosion in geomechanics applied to dams and levees (John Wiley \& Sons, 2013).

[2] J. King, I. Loveday, and R. L. Schuster, Quarterly Journal of Engineering Geology and Hydrogeology 22, 257 (1989).

[3] T. Kenney and D. Lau, Canadian Geotechnical Journal 22, 215 (1985)

[4] A. Skempton and J. Brogan, Geotechnique 44, 449 (1994).

[5] M. Li and R. J. Fannin, Canadian Geotechnical Journal 45, 1303 (2008).

[6] J. Sjah and E. Vincens, International Journal for Numerical and Analytical Methods in Geomechanics 37, 1231 (2013).

[7] E. Vincens, K. J. Witt, and U. Homberg, Acta Geotechnica 10, 291 (2015).

[8] W. Li, E. Vincens, N. Reboul, and B. Chareyre, in Scour and Erosion: Proceedings of the 7th International Conference on Scour and Erosion, Perth, Australia, 2-4 December 2014 (CRC Press, 2014) p. 241.

[9] T. Shire, C. O'Sullivan, K. Hanley, and R. Fannin, Jour- nal of Geotechnical and Geoenvironmental Engineering 140, 04014072 (2014).

[10] R. Jaeger, M. Mendoza, and H. J. Herrmann, Physical review letters 119, 124501 (2017).

[11] K. Kawano, T. Shire, and C. O'Sullivan, in EPJ Web of Conferences, Vol. 140 (EDP Sciences, 2017) p. 10005.

[12] R. Hill, Journal of the Mechanics and Physics of Solids 6, 236 (1958).

[13] F. Nicot, L. Sibille, and F. Darve, International Journal of Solids and Structures 46, 3938 (2009).

[14] F. Nicot, L. Sibille, and F. Darve, International Journal of Plasticity 29, 136 (2012).

[15] R. Wan, F. Nicot, and F. Darve, Failure in Geomaterials, a contemporary treatise (ISTE/Elsevier, 2016).

[16] N. Reboul, E. Vincens, and B. Cambou, Granular Matter 10, 457 (2008).

[17] C. O'Sullivan, J. Bluthé, K. Sejpar, T. Shire, and L. Cheung, Computers and Geotechnics 64, 120 (2015).

[18] A. Wautier, S. Bonelli, and F. Nicot, Granular Matter 19, 22 (2017).

[19] N. Mahabadi and J. Jang, Applied Physics Letters 110, 
041907 (2017).

[20] R. Beguin, P. Philippe, and Y.-H. Faure, Journal of Hydraulic Engineering 139, 1 (2012).

[21] A. Wautier, S. Bonelli, and F. Nicot, "Rattlers' contribution to granular plasticity and mechanical stability." (2018), accepted in International Journal of Plasticity.

[22] B. Chareyre, A. Cortis, E. Catalano, and E. Barthélemy, Transport in porous media 94, 595 (2012).

[23] M. Robinson, M. Ramaioli, and S. Luding, International journal of multiphase flow 59, 121 (2014).

[24] A. Drescher and G. D. J. De Jong, Journal of the Mechanics and Physics of Solids 20, 337 (1972).

[25] C. Liu, S. R. Nagel, D. Schecter, S. Coppersmith, S. Majumdar, et al., Science 269, 513 (1995).

[26] F. Radjai, D. E. Wolf, M. Jean, and J.-J. Moreau, Physical review letters 80, 61 (1998).

[27] S. Bardenhagen, J. Brackbill, and D. Sulsky, Physical Review E 62, 3882 (2000).

[28] J. F. Peters, M. Muthuswamy, J. Wibowo, and A. Tordesillas, Physical review E 72, 041307 (2005).

[29] A. Tordesillas, D. M. Walker, and Q. Lin, Physical Review E 81, 011302 (2010).

[30] B. Cambou, M. Jean, and F. Radjai, Micromechanics of granular materials (John Wiley \& Sons, 2013).

[31] P. A. Cundall and O. D. Strack, Geotechnique 29, 47
(1979).

[32] V. Šmilauer et al., Yade Documentation 2nd ed (The Yade Project, 2015) http://yade-dem.org/doc/.

[33] A. Wautier, S. Bonelli, and F. Nicot, in Book of extended abstracts: Multiscaled approaches towards degradation, damage and aging in geomaterials. (GDRI GeoMech, 2017) p. 82.

[34] A. Wautier, S. Bonelli, and F. Nicot, International Journal For Numerical and Analytical Methods in Geomechanics , 19 (2018).

[35] M. Rashidi, L. Peurrung, A. Tompson, and T. Kulp, Advances in Water Resources 19, 163 (1996).

[36] M. Johns, A. Sederman, A. Bramley, L. Gladden, and P. Alexander, AIChE journal 46, 2151 (2000).

[37] P. Magnico, Chemical engineering science 58, 5005 (2003).

[38] R. S. Maier, D. M. Kroll, H. T. Davis, and R. S. Bernard, Journal of colloid and interface science 217, 341 (1999).

[39] F. Nicot and F. Darve, International Journal of Solids and Structures 43, 3569 (2006).

[40] H. Zhu, F. Nicot, and F. Darve, International Journal of Solids and Structures 96, 25 (2016).

[41] F. Nicot, N. Hadda, and F. Darve, International Journal for Numerical and Analytical Methods in Geomechanics 37, 2987 (2013). 